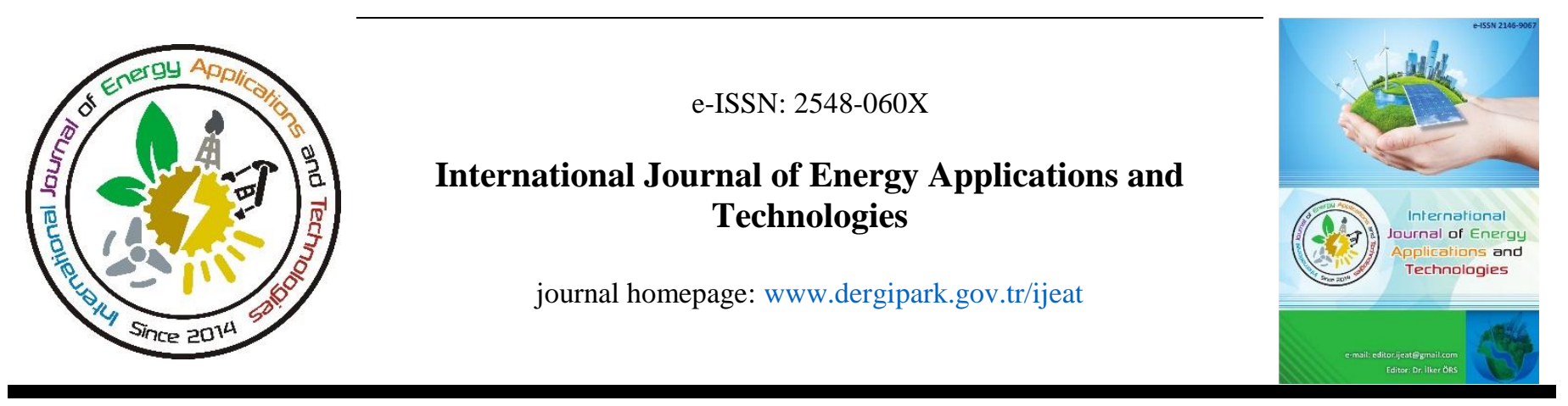

Original Research Article

\title{
Solar power plant generation forecasting using NARX neural network model: A case study
}

\author{
Obed Nkuriyingoma ${ }^{*}$, Saltuk Buğra Selçuklu ${ }^{2,3}$ \\ ${ }^{I}$ Department of Mechanical and Energy Engineering, School of Engineering, College of Science and Technology, University of Rwanda, Kigali, Rwanda \\ ${ }^{2}$ Energy Systems Engineering, Erciyes University, Kayseri, Turkey \\ ${ }^{3}$ Energy Conversion Research and Application Center, Erciyes University, Kayseri, Turkey
}

\author{
ARTICLE INFO \\ * Corresponding author \\ $\underline{\text { nkuliyobas@ gmail.com }}$ \\ Received January 29, 2021 \\ Accepted September 26, 2021 \\ Published by Editorial Board \\ Members of IJEAT \\ (C) This article is distributed by \\ Turk Journal Park System under \\ the CC 4.0 terms and conditions.
}

doi: 10.31593/ijeat. 870088

\begin{abstract}
New technologies have been developed and adopted to generate energy from renewable sources to satisfy the increasing demand without causing environmental damage. However, estimating the power output of inherently intermittent, weather-driven, and non-dispatchable renewable energy sources is a major scientific and societal concern. In this study, a neural network model to enable short-to-middle term forecasts of a photovoltaic (PV) power system is provided. Using historical weather and power generation data, a non-linear autoregressive network with exogenous input (NARX) model is built to forecast the non-linear photovoltaic system output. The performance of the model is then analyzed by different statistical evaluation parameters. It is shown that the PV system power output estimation method can be successfully employed.
\end{abstract}

Keywords: Artificial neural network; Energy generation forecast; NARX; Photovoltaic (PV); Solar energy

\section{Introduction}

The energy demand is increasing day by day as the world's population increases and technology advances. The primary energy sources for electric generation are natural gas, coal, nuclear, hydro, and renewables, such as solar, wind, and biomass. Coal, and natural gas have high shares compared to others but the transition towards renewable energy sources is gradually increasing [1]. New technologies and policies are implemented to increase the share of renewable energy sources in the production of electricity for environmental concerns and the reduction of greenhouse gas emissions. These technologies generate electricity from renewable energy sources such as solar and wind as efficiently as possible [2]. The greatest challenges that face these technologies are intermittency and variability.

Solar energy shows a high potential, and electricity generation from solar photovoltaic (PV) systems is constantly increasing. The output power of a solar PV plant is not constant due to its dependence on weather variables such as global solar radiation, air temperature, wind, relative humidity, air pressure, cloudiness, and dew point and also varies according to seasons of the year and time of the day. However, the forecasting of electricity generation from a solar PV plant is needed in operational planning, plant optimization, and for solar energy trading [3]. Additionally, due to the intermittency of solar energy, its integration into the utility grid is a big challenge $[4,5]$. To overcome this challenge, several tools are used to forecast solar PV plant output power.

Forecasting methods can be categorized into three different methods: physical, statistical, and hybrid methods. The physical method is based on the numerical weather prediction (NWP) or atmosphere by using physical data such as temperature, pressure, and cloud cover. The statistical model 
utilizes the historical data to train the models and is considered as a mathematical model. The hybrid method is a combination of physical and statistical approaches [6]. The choice of a forecasting method and model depends on the available information, needed resolution, accuracy, and purpose (very short-, short-, and long-term forecasting). Very short term or intra-day forecasting deals with forecasts up to 6 hours ahead. Short term forecasting provides predictions up to 7 days ahead. Long-term forecasting usually refers to annual forecasting and is necessary for strategic planning [7]. The numerical weather prediction is based on the physics of the atmosphere. The NWP uses current observations of the weather and processes this data to predict the future states of the weather using supercomputers. The NWP is good for one day to multi-days ahead horizon. The transient variations in clouds, which are considered the major obstacles for solar irradiance at the ground, are predicted in the NWP. To achieve high temporal and spatial resolution for intra-hour forecasts, NWP and satellite forecasts are currently inadequate. Ground observations using a sky imager present an opportunity to fill this forecasting gap and deliver a subkilometer view of cloud shadows over a large-scale PV power plant or an urban distribution feeder [8].

For physically-based forecasting, cloud cover and cloud optical depth are the most important parameters affecting solar irradiance [6]. The satellite and cloud imagery-based model is a physical forecasting model that analyzes clouds. The satellite and cloud imageries are processed to characterize clouds and detect their variability. This model works by determining the cloud structures during earlier recorded time steps. Understanding the structure of the clouds and their positions will help in predicting solar irradiance. Under low sun elevations, low irradiance conditions, and high spatial variability, the errors of satellite and cloud images can increase significantly [8].

The Autoregressive Moving Average (ARMA) model, also known as the Box-Jenkins model [9], is a type of time-series models in statistical methods. It consists of two parts. One is auto-regressive (AR) and the other is moving average (MA). The ARMA model is usually applied to auto-correlated time series data. ARMA models are very flexible since they can represent several different types of time series by using different orders. They have been proved to be competent in prediction when there is an underlying linear correlation structure in the time series. ARMA model has good performance in stationary time series and is a good tool for forecasting future values [9-11].

The Autoregressive Integrated Moving Average (ARIMA) model is another popular time series model used in the forecasting future value of a time series. The ARIMA model has three parts: the AR part, the MA part, and the integrated process (also known as the differentiating process). ARIMA models are used in non-stationary time series where they have to be transformed into stationary series by the integrated part of ARIMA. In an ARIMA model, the predicted future value of a time series is expressed as a linear combination of past values and past errors [11, 12].

Artificial Neural Networks (ANNs) are forecast models that utilize the idea of the biological neural system in the human brain for modeling complex real-world problems. ANNs are the foundation of Artificial Intelligence (AI) and solve problems that would prove impossible or difficult by human or statistical standards. An ANN has self-learning capabilities that enable it to produce better results as more data becomes available. ANN models are considered as nonlinear statistical models to understand the complex relationships between inputs and outputs and to find the patterns of data. The main characteristics of an ANN are nonlinearity, high parallelism, faults, and noise tolerance, and learning and generalization capabilities. ANNs are built like the human brain with neuron nodes interconnected.

ANNs have hundreds or thousands of artificial neurons called processing units which are interconnected by nodes. These processing units are made up of input and output units. The input units receive various forms and structures of information based on an internal weighting system and the neural network attempts to learn about the information presented in order to produce one output report. Generally, an ANN model consists of three layers: input, hidden, and output $[13,14]$. The input signals in neurons are multiplied by respective weights and then fed to the summing junction. Bias in the neuron is summed with the weighted inputs and the result is processed into activation function to produce the output as shown in Figure 1.

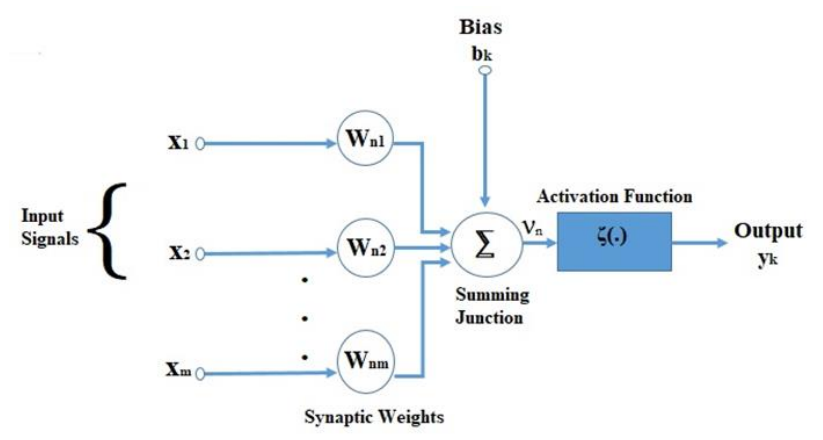

Fig. 1. ANN with $m$ inputs

The nonlinear autoregressive network with exogenous inputs (NARX) is a type of a recurrent ANN. In this study, a NARX model to enable short-to-middle term forecasts of the PV power system is provided and the performance of the model is analyzed for a power plant located in Kayseri, Turkey. Details of the NARX model is presented in the Methodology section. 
Accurate forecasting of power generated from a PV power plant is essential to meet the increasing energy demand, to mitigate climate change, and to stabilize electric grid systems, and it is considerably complex because of its dependence on the uncertain weather and climate factors. The indirect forecasting models forecast the solar irradiance by using various methods, including NWP, image-based, statistical, and ANN-based methods [4, 15-19]. The forecasted solar irradiance data become the input to commercial PV simulation software such as a hybrid optimization model for electric renewables (HOMER), and transient systems simulation (TRNSYS) to forecast the PV power generation [2]. The direct forecasting models predict PV power plant output using historical weather data $[1,5,20-$ 25]. Module temperature and weather parameters such as wind speed and direction, humidity, atmospheric temperature, and solar irradiance are considered as potential parameters for forecasting the PV system output power [2]. Ahmad, Anderson, and Lie [4] investigated several models for hourly solar irradiation forecasting that can be implemented into a future solar control system, and they emphasized the ability of NARX network. NARX model appears to perform better in short and long-term forecasting compared to other investigated models, such as Multilayer Perceptron (MLP) neural network, ARMA, and persistence models. The generalization capability and ability of NARX model to produce accurate predictions were demonstrated by the obtained results. Forecasting ability of NARX model was shown by Cadenas et al. [26]. In the comparative research on wind speed prediction, more accurate results were obtained by using the NARX model compared to the ARIMA model. NARX model can be used to predict solar irradiation and wind speed as well. DiPiazza et al. [27] present a study on solar and wind forecasting by using NARX neural networks. The hourly solar irradiation and wind speed were forecasted by the NARX network model for several time horizons in a range of 8 to 24 hours ahead. It was found that the model can be implemented within a smart grid management system. In the literature, ANN models such as MLP, radial basis function (RBF), and NARX were proposed for solar radiation forecast. Al Shamisi et al. [15] worked on the use of MATLAB to develop artificial neural network models for predicting global solar radiation in Al Ain City, UAE, and it was found out that ANN models have good performance in general. Sandhya and Kavitha [28] proposed a neural network-based approach to predict solar radiation. It was found that the NARX network trained with the LevenbergMarquardt algorithm converges faster than other algorithms and that can recognize the relationship between the input and output variables and predict solar radiation accurately and produces more accurate results than the feed-forward neural network models. Ahmad and Anderson [29] proposed solar radiation prediction using an artificial neural network model for New Zealand. Considering the impact of each weather parameter on global solar radiation, the prediction accuracy of the proposed ANN was found to be dependent on various combinations of weather variables, and a NARX recurrent dynamic network model for global solar irradiance prediction was developed. Mohammed et al. [17] studied hourly solar radiation prediction based on NARX neural network to investigate the ability to predict hourly solar radiation in Amman, Jordan. The results showed that the NARX neural network model trained with the Levenberg-Marquardt algorithm converges faster.

For direct solar forecast, which consists of PV output power predictions, more models were investigated and studied. Das et al. [2] review PV power forecasting models and model optimization studies. The persistence models that project solar irradiance to future time steps were used for predicting simple and complex PV power system. ANN-based models perform better than conventional mathematical models in terms of accuracy and adaptability. The fuzzy logic (FL) method was used for medium-term forecasting models because of its ability of handling the uncertainty of real-world data. FL method combined with a neural network produces an adaptive network-based fuzzy inference system (ANFIS) method and results in a $95 \%$ improvement of accuracy. Support vector machine (SVM) models, unlike ANN, have the ability to learn without depending on prior knowledge. For hybrid models, the input data were preprocessed by wavelet transform (WT) selected by the fuzzy system or kmeans clustering and smoothed by WT before being applied to the forecasting models. In hybrid models, the performance is improved by combining the advantages of the individual model. Kardakos et al. [13] studied the application of time series and ANN models in the short-term forecasting of PV power generation. The forecasting models were applied to different PV plants in the Greek power systems and the simulation results were presented in their work. The used forecasting models were seasonal ARIMA (SARIMA) time series that was further improved by incorporating short-term solar radiation forecasts derived from NWP models and ANN with multiple inputs. Modified SARIMA and ANN models are preferable since they lead to considerably improved dayahead forecasts. Cai et al. [30] studied forecasting power output for a grid-connected PV power system without using solar radiation measurement. A novel PV power forecasting method based on the adaptive NARX network model was proposed. The data used as input were calculated future hourly values of solar radiation under the clear-sky condition. Furthermore, to characterize solar radiation on rainy days adopted online weather forecast data from a public website were used as the remaining input of the forecast model. The results prove the ability of NARX model to forecast the 
hourly power output of the PV power system precisely. Sharma et al. [24] proposed a system for the prediction of output solar power generation using the neural network time series method. The developed ANN model was based on NARX. The network was trained by the LevenbergMarquardt algorithm, and the simulation results were claimed to be effective along with a very high level of accuracy. Table 1 provides a summary of the reviewed papers.
Considering the advantages provided in the literature, we establish a NARX model to forecast the non-linear photovoltaic system output using historical weather and power generation data. The structure of the paper is as follows: the next section provides the details of the methodology. The third section presents the results and discussions, and the fourth section concludes the paper.

Table 1. Literature review summary

\begin{tabular}{|c|c|c|c|c|}
\hline Authors & Forecasting model & Target Variable & Forecasting error & $\begin{array}{l}\text { Forecasting } \\
\text { horizon }\end{array}$ \\
\hline Cadenas et al. (2016) & $\begin{array}{l}\text { ARMA } \\
\text { NARX }\end{array}$ & Wind speed & $\begin{array}{l}\text { MAE } 5.5 \% \text { for hourly database and } 2.3 \% \text { for } \\
\text { minute database } \\
\text { MSE } 10.6 \% \text { for hourly database and } 12.8 \% \text { for ten- } \\
\text { minute database }\end{array}$ & $\begin{array}{l}10 \text { min ahead } \\
1 \mathrm{~h} \text { ahead }\end{array}$ \\
\hline Al Shamisi et al. (2010) & ANN (RBF\&MLP) & Solar radiation & $\begin{array}{l}\text { Coefficient of determination } 0.80-0.93 \text {, RMSE } \\
0.349-0.616 \text { and MBE }\end{array}$ & 1 month ahead \\
\hline $\begin{array}{l}\text { Sandhya and Kavitha } \\
\text { (2015) }\end{array}$ & NARX & Solar radiation & MSE $1.5-6$ and Regression Analysis $0.16-0.78$ & - \\
\hline $\begin{array}{l}\text { Ahmad and Anderson } \\
\text { (2014) }\end{array}$ & NARX & $\begin{array}{l}\text { Global solar } \\
\text { radiation }\end{array}$ & Regression (R) 0.949-0.963, MSE 0.0591-0.0722 & $24 \mathrm{~h}$ ahead \\
\hline Ahmad et al. (2014) & NARX & $\begin{array}{l}\text { Global solar } \\
\text { radiation }\end{array}$ & $\begin{array}{l}\text { RMSE } 0.243-0.280 \mathrm{MJ} / \mathrm{m}^{2} \text {. Regression (R) 0.949- } \\
0.963\end{array}$ & $24 \mathrm{~h}$ ahead \\
\hline Mohammed et al. (2013) & NARX & $\begin{array}{l}\text { Global solar } \\
\text { radiation }\end{array}$ & $\begin{array}{l}\text { Coefficient of determination (R) } 0.98692- \\
0.99157 \text {, RMSE } 42.8367-53.2732 \mathrm{MJ} / \mathrm{m}^{2} / \text { hour, } \\
\text { MBE 25.5612-32.5375 MJ } / \mathrm{m}^{2} / \text { hour }\end{array}$ & $24 \mathrm{~h}$ ahead \\
\hline Di Piazza et al (2016) & NARX ANN & $\begin{array}{l}\text { Global solar } \\
\text { radiation and } \\
\text { wind speed }\end{array}$ & $\begin{array}{l}\text { Normalized RMSE } 0.260-0.588 \text {, Coefficient of } \\
\text { variation of RMSE } 0.635-3.098\end{array}$ & 8 to $24 \mathrm{~h}$ ahead \\
\hline Kardakos et al. (2013) & $\begin{array}{l}\text { ANN } \\
\text { Seasonal ARIMA }\end{array}$ & PV output power & Normalized RMSE 3,17- 20,35 & $1 \mathrm{~d}$ ahead \\
\hline Cai et al. (2010) & $\begin{array}{l}\text { NARX ANN } \\
\text { MLP ANN }\end{array}$ & PV output power & MAPE $16.47 \%-30.72 \%$ & Not specified \\
\hline Sharma et al. (2016) & NARX ANN & PV output power & MSE 7.5 & $24 \mathrm{~h}$ ahead \\
\hline Das et al. (2018) & $\begin{array}{l}\text { Persistence } \\
\text { ARIMA } \\
\text { ANN } \\
\text { SVM } \\
\text { Hybrid }\end{array}$ & PV output power & $\begin{array}{l}\text { Normalized MAE (NMAE) }<1 \text { to } 19.49 \% \text {, and } \\
\text { weighted MAE (WMAE) }<2 \text {, nRMSE } 2.74- \\
36.9 \% \text {, MAPE 2.38-18.89\%, MRE } 8.64-17.58 \% \text {, } \\
\text { MSE 21.8, MSE } 2.7-19.663 \% \text {, MAE } 3.38-21.02 \%\end{array}$ & $1 \mathrm{~min}$ to $24 \mathrm{~h}$ ahead \\
\hline
\end{tabular}

\section{Methodology and Model Design}

In this section, we first present the details of the NARX model. Transfer function and performance evaluation methods are discussed separately, and then, data collection, input data selection, and the generated NARX model are explained.

\subsection{Non-linear autoregressive network with exogenous} input

The nonlinear autoregressive network with exogenous inputs (NARX) is a type of recurrent ANN. In the NARX network, the feedforward network receives two inputs, exogenous inputs, and feedback from the output layer. The NARX model terminology is based on the linear ARX model, which is commonly used in time-series modeling. The defining equation for the NARX model is as follows:

$$
\begin{aligned}
& y(t)=f\left(y(t-1), y(t-2), \ldots, y\left(t-n_{y}\right), u(t-1), u(t-\right. \\
& \left.2), \ldots, u\left(t-n_{u}\right)\right)+e(t)
\end{aligned}
$$


Where $y(t)$ is the target output and $y(t-1), \ldots ., y\left(t-n_{y}\right), u(t-1)$, $\ldots, u\left(t-n_{u}\right)$ are past values of target output and external input at discreet time $t$, while $n_{y} \geq 1, n_{u} \geq 1$, and $n_{u} \leq n_{y}$ are the inputmemory (input delay) and output-memory (output delay) orders, and $e(t)$ is a noise term, generally assumed as Gaussian and white noise. The nonlinear mapping function $f($.) is generally unknown and can be approximated. The resulting connectionist architecture is then called a NARX network. NARX model is well suited for modeling nonlinear dynamic behaviors and has been extensively used in various applications. The output $y(t)$ is regressed on previous values of the output and previous values of input. NARX network can be implemented by using feedforward neural network architecture to approximate the function $f($. $)[2,26,27,30] .0$ 2 shows the architecture of the NARX network.

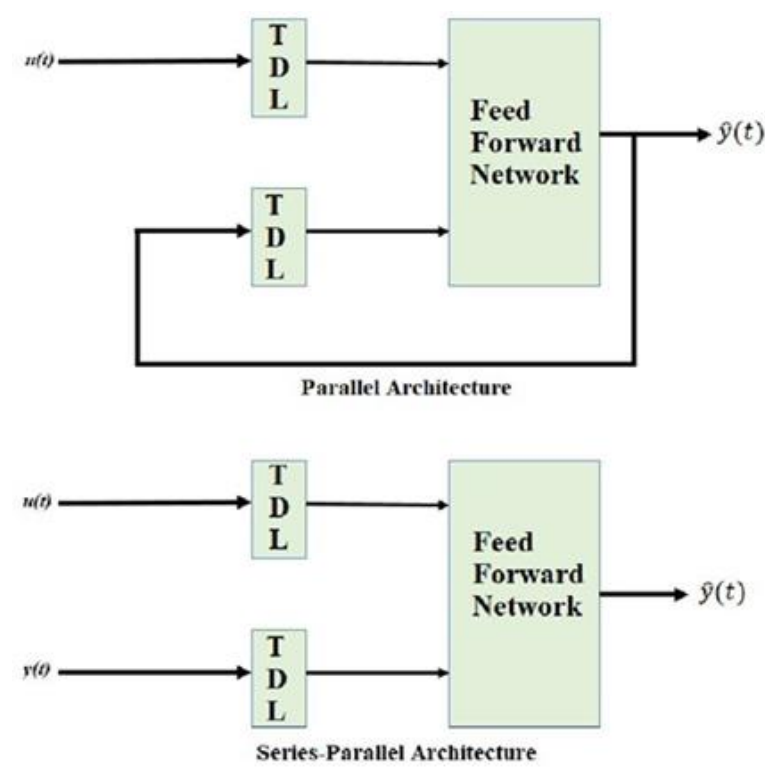

Fig. 2. NARX architecture (TDL: tapped delay lines)

\subsection{Transfer function}

Transfer function, also known as the activation function, controls the amplitude of the output of the neural network model. The transfer function translates the input signals to output signals. The commonly used transfer functions are sigmoid (logistic and hyperbolic tangent), linear, and Gaussian as shown in Figure 3.

The sigmoid (logistic and hyperbolic tangent) function is the most used among others due to the simplicity in finding its derivative. Usually, these functions are applied in the hidden layer of the network $[15,31]$.

\begin{tabular}{|c|c|c|}
\hline Function name & Mathematical equation & Graphical illustration \\
\hline Linear & $f(x)=x$ & \\
\hline $\begin{array}{c}\text { Hyperbolic } \\
\text { Tangent Sigmoid }\end{array}$ & $f(x)=\frac{e^{x}-e^{-x}}{e^{x}+e^{-x}}$ & \\
\hline Logistic Sigmoid & $f(x)=\frac{1}{1+e^{-x}}$ & \\
\hline Gaussian & $f(x)=\frac{1}{\sqrt{2 \pi \sigma}} e^{\frac{-(x-\mu)^{2}}{2 \sigma^{2}}}$ & \\
& & \\
\hline
\end{tabular}

Fig. 3. MATLAB Built-in Transfer Functions

\subsection{Performance evaluation}

To evaluate the performance of the developed models quantitatively, statistical analysis has to be conducted [15]. The accurate measurement of the PV power forecasting model is a vital part of the forecasting process [2]. Statistical evaluation parameters such as normalized root mean square error (nRMSE), mean bias error (MBE), mean absolute error (MAE), and mean absolute percentage error (MAPE) can be used to evaluate the performance of the forecasting model. nRMSE provides information on the short-term performance which is a measure of the variation of predicted values around the measured values. The lower the nRMSE, the more accurate is the estimation. MBE is an indication of the average deviation of the predicted values from the corresponding measured values and can provide information on the long-term performance of the models. Lower MBE values suggest a better long-term model prediction. A positive MBE indicates the amount of overestimation, and a negative MBE indicates the underestimation.

MAPE is commonly used in the performance evaluation of forecasting models. The interpretation of MAPE indicates the accuracy of the model. Less than $10 \%$ is a highly accurate forecast, 11 to $20 \%$ is a good forecast, 21 to $50 \%$ is a reasonable forecast, and $51 \%$ or more is an inaccurate forecast.

The absolute error is the absolute value of the difference between the forecasted value and the actual value. MAE shows the magnitude of an error we can expect from the forecast on average [15]. The expressions for the aforementioned statistical parameters are given by equations (2), (3), (4), and (5). 


$$
\begin{aligned}
& n R M S E=\frac{1}{N} \sqrt{\sum_{i=1}^{N}\left(W_{f}-W_{t}\right)^{2}} \times \frac{100}{W_{t \max }} \\
& M B E=\frac{1}{N} \sum_{i=N}^{N}\left(W_{f}-W_{t}\right) \\
& M A P E=\frac{1}{N} \sum_{i=1}^{N} \frac{\left|W_{f}-W_{t}\right|}{W_{t}} \times 100 \% \\
& M A E=\frac{1}{N} \sum_{i=1}^{N}\left|W_{f}-W_{t}\right|
\end{aligned}
$$

Where, $W_{f}, W_{t}$, and $W_{\text {tmax }}$ represent the forecasted values at each time point, the observed/measured values at each time point, and the maximum observed values, respectively. $N$ is the number of data samples for the time scale.

\subsection{Data collection and preprocessing}

In solar energy systems, the amount of produced power from a solar PV power plant depends on various factors. Variables that have an impact on solar energy are weather parameters and time of the day. In the proposed model weather parameters have been used as dependent variables for the forecasting of power that will be produced by a solar PV power plant. In addition, the hour angle and solar zenith angle of the location under consideration have been calculated and added as inputs to the model.

The considered solar plant is located in the Bünyan district in Kayseri province to the geographical coordinates of 38 40'29.84" N and 3600'38.49” E (Figure 4). Meteorological data was collected form Bünyan and Pınarbaş1 (a nearby district) meteorological stations under the authority of Ministry of Forestry and Water Management Meteorology General Directorate. The collected meteorological data is used as input to the model. Data of produced power by the solar PV power plant is provided by the company in charge of the plant (Hunat Energy) and used as the target data.

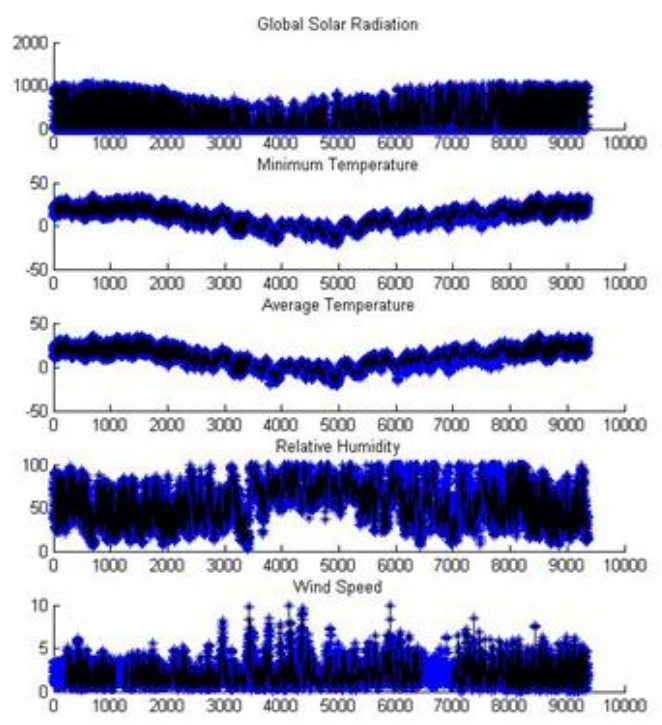

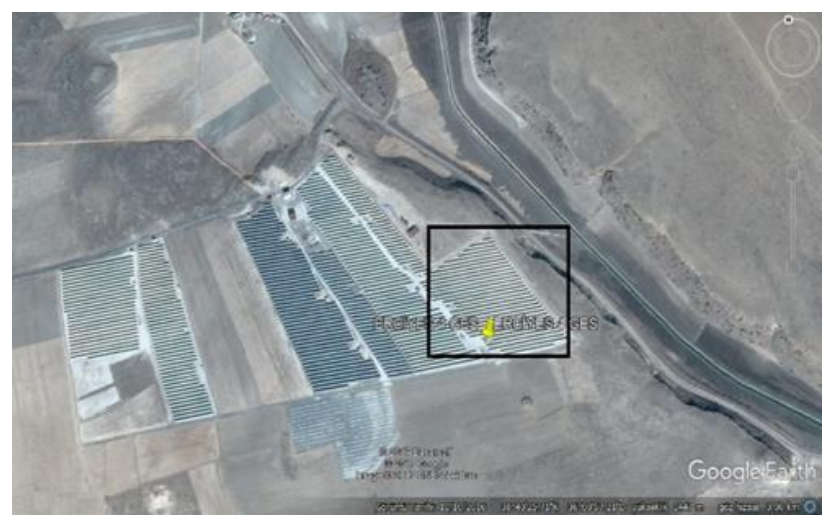

Fig. 4. Solar PV power plant location

The collected data cover the period of June 2016 to August 2017 for meteorological data, and June 2016 to July 2017 for solar PV power plant data. Meteorological data in the model are hourly global solar radiation, sunshine intensity, hourly temperature, minimum temperature, maximum temperature, wind speed, relative humidity, air pressure, and precipitation. Table 2 shows a list of collected variables together with their abbreviations and units.

Using Excel and MATLAB software, data is arranged into one table in which columns consist of meteorological variables, hour angle, zenith angle, and historical data of produced power by solar PV plant. To be accurate, meteorological data and power data are synchronized with respect to sunrise and sunset. The missing part of the data needs to be considered because they contain important information that may contribute to the performance of the model. In this study, missing data is filled using the Spline interpolation method of MATLAB due to its superiority in measure of smoothness compared to linear, nearest neighbor, and shape-preserving methods. Figure 5 shows data obtained from the meteorological station and interpolated data.
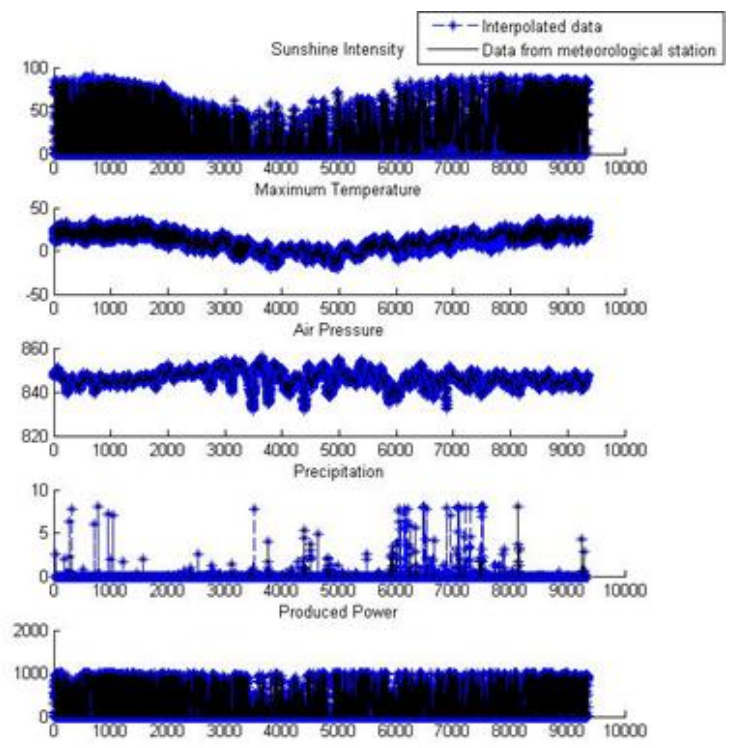

Fig. 5. Collected data and filling in missing data 
All collected data must be within the same range to increase the performance of the model. Therefore, input data is normalized in the range of 0 and 1 . The normalization is processed as follows:

$X^{\prime}=\frac{X-X_{\min }}{X_{\max }-X_{\min }}$

where $X^{\prime}$ is the normalized value, $X$ is the actual value, and $X_{\min }$ and $X_{\max }$ are the minimum and maximum values in the corresponding data column, respectively.

\subsection{Input data selection}

One of the major tasks in time series prediction is the selection of input variables. The selection of input variables plays a crucial role when modeling time series. When a group of independent variables is available, variable selection has to be performed in order to avoid variables that have little influence on the forecast and sometimes not to neglect useful ones. The relationship between the input and target variables can be linear, quadratic, inverse, and sine wave. The choice of variables depends on data availability, quality, and correlation with the target variable [15].
To identify the relationship between collected meteorological variables and solar power plant output, a statistical analysis is performed. The regression value $(\mathrm{R})$ is a measure of the linear correlation between two variables, giving a value between 1 and -1 , where 1 is a total positive correlation, 0 is no correlation, and -1 is a total negative correlation. The $\mathrm{R}$ values for each variable with respect to solar power output are shown in Table 2.

Table 2. List of collected data parameters and regression values

\begin{tabular}{llccc}
\hline & Variable & Abbreviation & Unit & $\mathrm{R}$ \\
\hline 1 & Produced Solar Power & OUTP & $k W$ & \\
2 & Global Solar Radiation & GSR & $W h / \mathrm{m}^{2}$ & 0.878 \\
3 & Sunshine Intensity & SSI & $\mathrm{Cal} / \mathrm{cm}^{2}$ & 0.878 \\
4 & Hourly Temperature & TMP & $\mathrm{C}$ & 0.270 \\
5 & Minimum Temperature & MNT & $C$ & 0.264 \\
6 & Maximum Temperature & MXT & $C$ & 0.278 \\
7 & Wind Speed & WDS & $\mathrm{m} / \mathrm{s}$ & 0.067 \\
8 & Relative Humidity & RLH & $\%$ & -0.406 \\
9 & Air Pressure & PRS & $h P a$ & 0.232 \\
10 & Precipitation & PRC & $\mathrm{mm}$ & -0.055 \\
& & & &
\end{tabular}

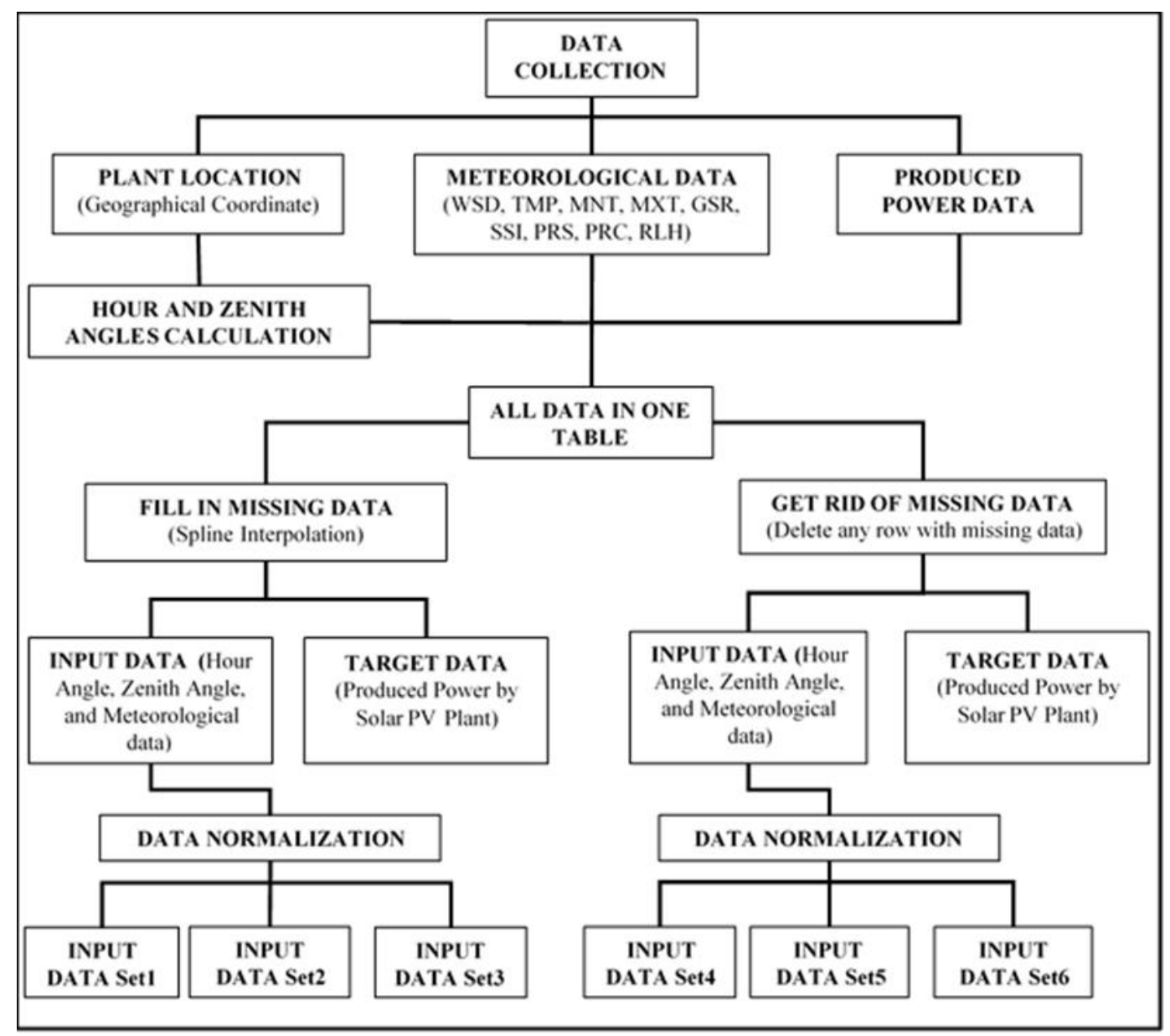

Fig. 6. Flow chart of data collection and processing 
From Table 2, it can be seen that global solar radiation, sunshine intensity, hourly temperature, minimum temperature, maximum temperature, relative humidity, and air pressure have reasonable degrees of correlation with solar PV plant output, while wind speed and precipitation have a very small correlation. Once the significant variables are determined (based on the regression values), three significant combinations of the nine meteorological variables as shown in Table 3 are chosen and each combination is used twice, first, filling in for missing values, and second, without filling in for missing values. In total, six different data sets are used as input to the model. Figure 6 shows data sets and how data is collected and processed.

Table 3. Input data set based on different combinations of input variables

\begin{tabular}{|l|}
\hline Data Sets with filling in for missing values \\
\hline HA, ZA, MNT, MXT, TMP, PRS, RLH, SSI, GSR, WDS, PRC \\
HA, ZA, MNT, MXT, TMP, PRS, RLH, SSI, GSR, WDS \\
HA, ZA, MNT, MXT, TMP, PRS, RLH, SSI, GSR \\
\hline Data Sets without filling in for missing values \\
\hline HA, ZA, MNT, MXT, TMP, PRS, RLH, SSI, GSR, WDS, PRC \\
HA, ZA, MNT, MXT, TMP, PRS, RLH, SSI, GSR, WDS \\
HA, ZA, MNT, MXT, TMP, PRS, RLH, SSI, GSR \\
\hline
\end{tabular}

\subsection{Generated NARX network model}

NARX network model is used to perform multistep ahead prediction using past data through learning. NARX neural network architecture is composed of three layers, that is, input, hidden, and output. The input layer receives different input information, the hidden layer, which may consist of a single or several layers, analyzes the input information, and the output layer receives the analyzed results and provides the output.

In the input layer, the number of neurons equals the number of inputs, and the number of neurons in the output layer is one. The number of neurons in hidden layer and number of input delays and feedback delay in tapped delay lines (TDL) are determined by the performance of the network, only initial values are provided to start training. As the network learns with trial and error, different cases must be considered to get the best performance. Therefore, the effect of changing the number of neurons in the hidden layer and increasing and decreasing the number of delays are also investigated. The network parameters are determined according to the network architecture and model structure. In series-parallel architecture (open-loop), the actual output data is used for network training, and in parallel architecture (closed-loop), the predicted output is fed back to the feedforward network thought tapped delay line (TDL). Table 4 shows parameters of the generated NARX network and Figure 7 shows the open-loop and closed-loop structure of the NARX network in MATLAB.
Table 4. Designed NARX model parameters

\begin{tabular}{|l|l|}
\hline $\begin{array}{l}\text { Dividing } \\
\text { Data }\end{array}$ & $\begin{array}{l}\text { Dividing Function: Dividrand } \\
\text { Training Ratio: 70\% } \\
\text { Validation Ratio: 15\% } \\
\text { Testing Ratio:15\% }\end{array}$ \\
\hline $\begin{array}{l}\text { Network } \\
\text { Architecture }\end{array}$ & $\begin{array}{l}\text { Input Delay: 4 } \\
\text { Feedback Delay: 4 } \\
\text { Number of Hidden Neurons: From 50 to 150 }\end{array}$ \\
\hline $\begin{array}{l}\text { Training } \\
\text { Algorithm }\end{array}$ & Levenberg-Marquardt backpropagation (trainlm) \\
\hline $\begin{array}{l}\text { Performance } \\
\text { Function }\end{array}$ & Mean squared error and Mean absolute error \\
\hline $\begin{array}{l}\text { Plot } \\
\text { Functions }\end{array}$ & $\begin{array}{l}\text { Performance, train state, error histogram, } \\
\text { regression, response, error correlation, input error } \\
\text { correlation }\end{array}$ \\
\hline $\begin{array}{l}\text { Transfer } \\
\text { Function }\end{array}$ & $\begin{array}{l}\text { Hidden Layer: Hyperbolic tangent sigmoid } \\
\text { Output Layer: Hyperbolic tangent sigmoid }\end{array}$ \\
\hline & $\begin{array}{l}\text { Open Loop Network: Stops if MAE is less or equal } \\
\text { than the minimum goal } \\
\text { Closed-Loop Network: Stats after open-loop } \\
\text { Netwing } \\
\text { training and stops if MAE is less than or equals to } \\
\text { the MAE of open-loop network }\end{array}$ \\
\hline
\end{tabular}
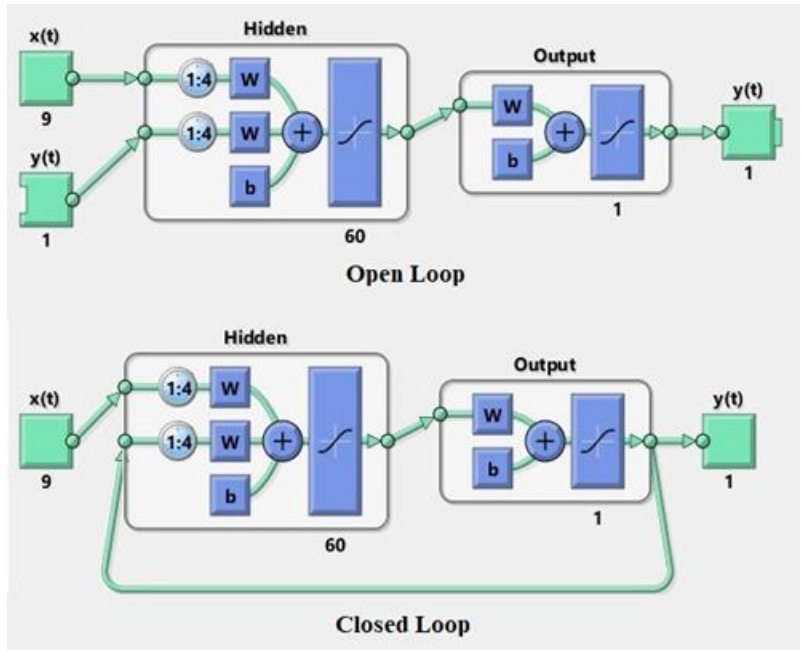

Fig. 7. NARX network

\section{Results and Discussion}

We present results in three sections for network training, network model simulation, and performance evaluation of the model.

\subsection{NARX network training}

The model is trained in two successive steps. The first step is to train the NARX network in open-loop mode. The second step is to train the NARX network in closed-loop mode. Open-loop training of the network helps to find the number of hidden neurons for the best possible performance. For our case, the minimum performance goals are set to MAE of 53 $\mathrm{kW}$ for data set 1 to data set 3 , and $75 \mathrm{~kW}$ for data set 4 to data set 6 . These values are selected after several trials. The open-loop training stops if the MAE is less than or equal to the performance goal. In addition, all of the training is done in the open-loop network including the validation and testing steps. 
In open-loop training of the network, the number of input and feedback delays are set to four and kept constant. On the other hand, the number of hidden neurons varies. The initial number of hidden neurons is set to 50. The performance of the network and its behaviors can be visualized with the help of different figures that show the network response during training. Figure 8, Figure 9, and Figure 10 are regression, training state, validation performance, respectively. Figure 8 shows the regression between target outputs and predicted values and gives the estimation linear equation and regression value between targets and predicted values. The training states of the neural network during the epoch at which the target is reached are shown in Figure 9. Figure 10 indicates the best validation performance of the neural network and shows training, validation, and test curves of the training process for the goal set in each case and the number of epochs (training iterations) at which the goal has been reached. It indicates how the gradient and weights change and the number of validation checks during the epochs of the neural network training process. MU is the training gain that controls the change of weights on each iteration. These

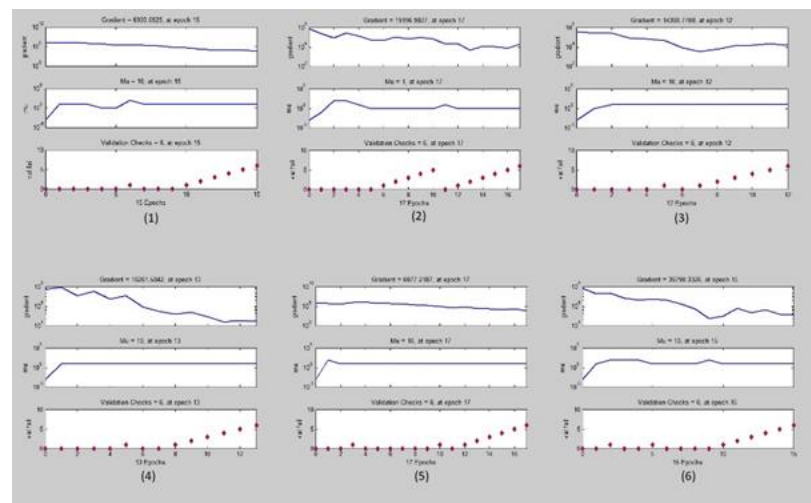

Fig. 9. Training state outputs of the training process indicate a successful state of the NARX neural network. Table 5 is a summary of the training performance. It can be found from Table 5 that the best performance, in general, is obtained by data set 3 , where input data set is filled in for missing data, and wind speed and precipitation data are not used.

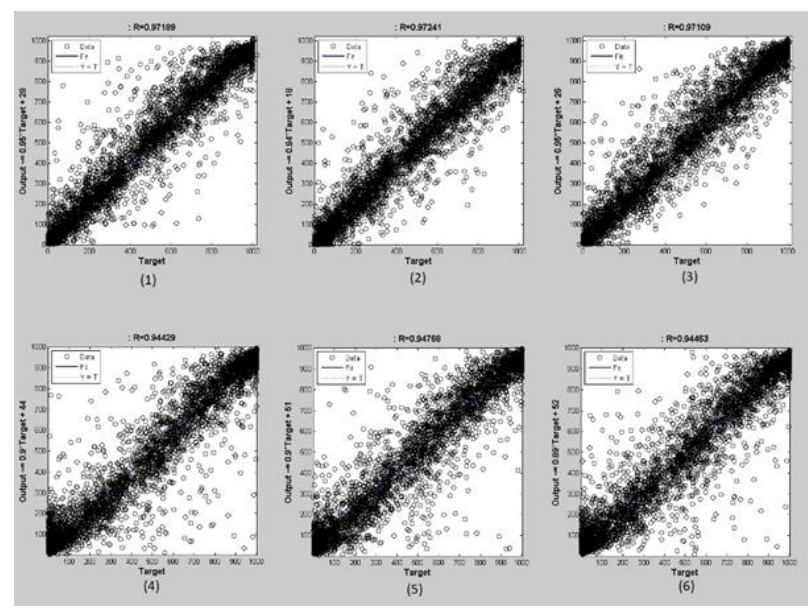

Fig. 8. Regression performance

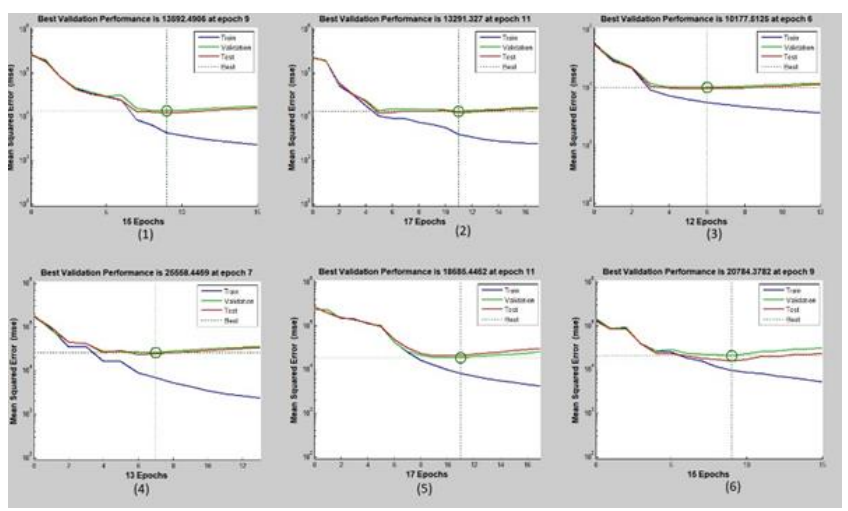

Fig. 10. Validation performance

Table 5. Training performance summary

\begin{tabular}{lcccccc}
\hline Data Set & $\mathbf{1}$ & $\mathbf{2}$ & $\mathbf{3}$ & $\mathbf{4}$ & $\mathbf{5}$ & $\mathbf{6}$ \\
\hline Hidden Neuron & 114 & 52 & 60 & 97 & 79 & 74 \\
MAE Open Loop & 52.6 & 51.8 & 52.6 & 72.3 & 72.2 & 73.7 \\
MAE Closed Loop & 47.1 & 50.1 & 49.5 & 67.6 & 68.6 & 72.2 \\
MSE Open Loop & 6862 & 6595.9 & 6851.8 & 12285.8 & 11530.7 & 12177.9 \\
MSE Closed Loop & 5537.9 & 6143.2 & 5967.4 & 10714.7 & 10402.2 & 11656.8 \\
RMSE Open Loop & 82.8 & 81.2 & 82.8 & 110.8 & 107.4 & 110.3 \\
RMSE Closed Loop & 74.4 & 78.4 & 77.2 & 103.5 & 102 & 108 \\
R & 0.9719 & 0.9724 & 0.9711 & 0.9443 & 0.9477 & 0.9446 \\
Best Validation Performance & 13592.5 & 13291.3 & 10177.5 & 25558.4 & 18685.4 & 20784.4 \\
$\boldsymbol{N}^{\text {th }}$ Epoch & 9 & 11 & 6 & 7 & 11 & 9 \\
Gradient & 6909 & 19197 & 14308.8 & 16261.5 & 6077.2 & 36790.3 \\
Mu & 10 & 1 & 10 & 10 & 10 & 10 \\
Validation Check & 6 & 6 & 6 & 6 & 6 & 6 \\
Number of Epoch & 15 & 17 & 12 & 13 & 17 & 15 \\
Computational Time & $338 \mathrm{~min}$ & $145 \mathrm{~min}$ & $611 \mathrm{~min}$ & $85 \mathrm{~min}$ & $286 \mathrm{~min}$ & $87 \mathrm{~min}$ \\
\hline
\end{tabular}




\subsection{NARX network model simulation}

NARX network model is generated in MATLAB/Simulink. Figure 11 (a) and (b) show the external appearance of the model and internal architecture, respectively. Layer 1 and Layer 2 in Figure 11 (b) represent the network hidden layer and output layer. The output of the hidden layer is fed to the input of the output layer, and the output of the output layer is feedback to the hidden layer.

The model is used to forecast the output of the solar PV plant from the $1^{\text {st }}$ of August to the $8^{\text {th }}$ of August 2017. The forecasted values are on an hourly basis and aggregated on a daily basis using the averaging method. Figure 12 shows the forecasted and real generated power from the solar PV plant for six data sets.

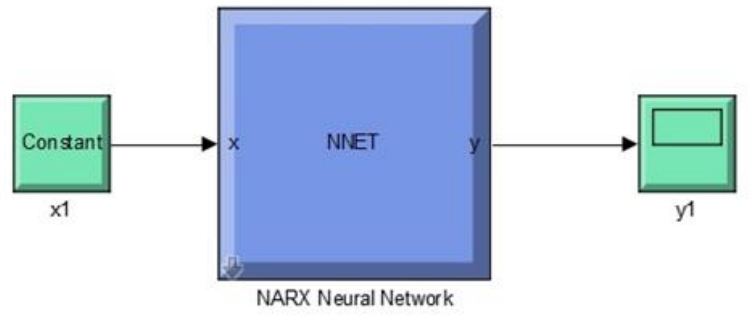

(a)

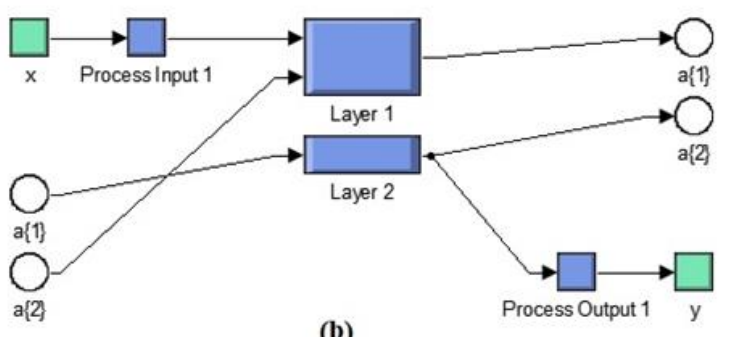

Fig. 11. NARX network model in MATLAB/Simulink

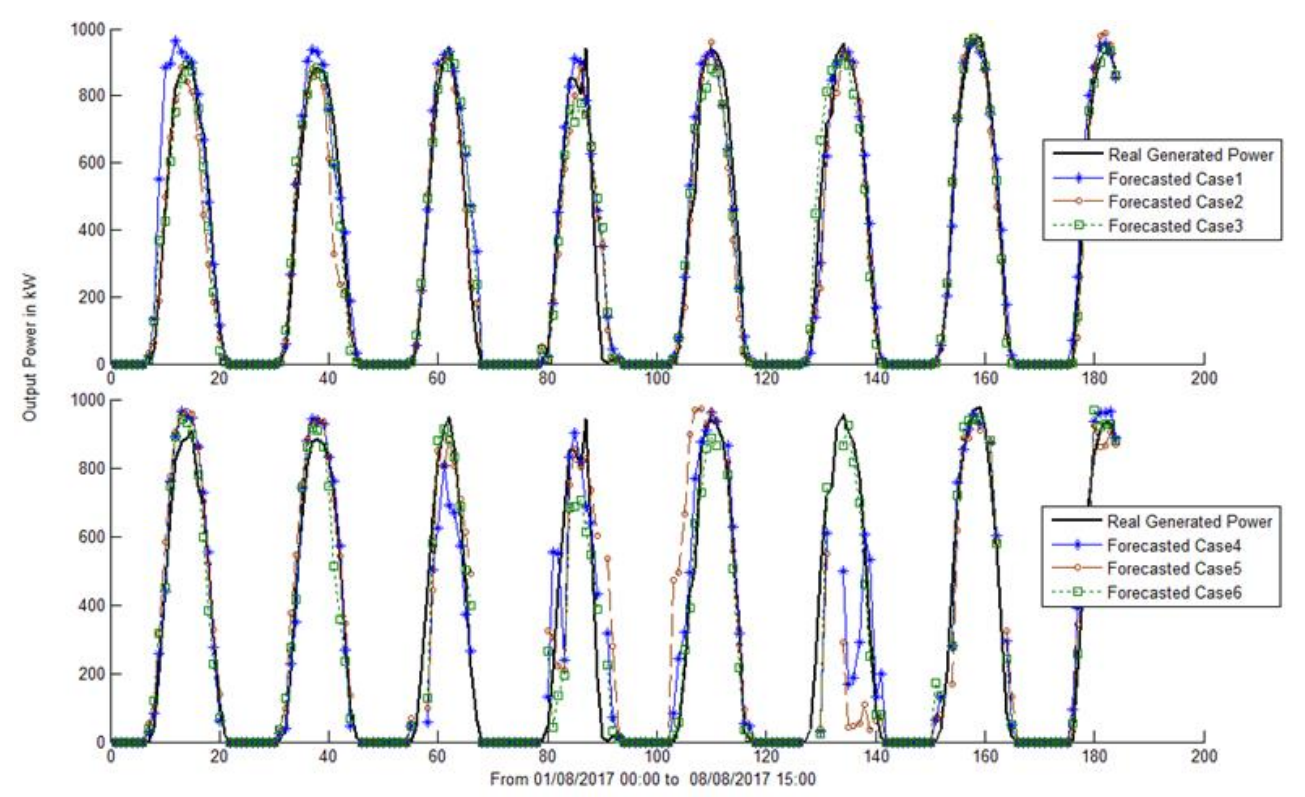

Fig. 12. Hourly forecasted and expected output power

\subsection{Model performance evaluation}

The model performance was evaluated using MAE, MAPE, MBE, and nRMSE. Tables 6 and 7 summarize hourly and daily forecast errors, respectively. For the hourly forecast, data set 3 has the smallest errors for MAE, MAPE, and nRMSE and data set 2 has the smallest errors for MBE. For the daily forecast, the performance of data set 2 and 3 is almost the same with the smallest errors for MAE, MAPE, and MBE, and nRMSE. Table 8 is a comparison of the obtained results with other works in the literature review and shows that the designed NARX model performs as good as or better than others and can be successfully implemented for PV power output forecast.
Table 6. Hourly forecasting errors for all cases

\begin{tabular}{ccccc}
\hline Case & MAE & MAPE & MBE & nRMSE \\
\hline 1 & 66.489 & $17.93 \%$ & -1.468 & $10.27 \%$ \\
2 & 68.445 & $15.39 \%$ & -0.0242 & $10.49 \%$ \\
3 & 52.0815 & $11.83 \%$ & 8.253 & $8.57 \%$ \\
4 & 113.001 & $29.67 \%$ & -8.0138 & $18.43 \%$ \\
5 & 153.835 & $39.13 \%$ & 4.434 & $24.99 \%$ \\
6 & 86.0227 & $20.53 \%$ & -19.354 & $12.83 \%$
\end{tabular}

Table 7. Daily forecasting errors for all cases

\begin{tabular}{ccccc}
\hline Case & MAE & MAPE & MBE & nRMSE \\
\hline 1 & 44.492 & $9.05 \%$ & -4.181 & $8.44 \%$ \\
2 & 17.396 & $3.59 \%$ & 1.0163 & $3.59 \%$ \\
3 & 20.858 & $4.60 \%$ & 9.206 & $4.53 \%$ \\
4 & 80.078 & $14.47 \%$ & -16.818 & $15.98 \%$ \\
5 & 106.077 & $20.08 \%$ & -12.621 & $25.73 \%$ \\
6 & 34.748 & $6.69 \%$ & -18.929 & $6.63 \%$ \\
\hline
\end{tabular}


Table 8. Results Comparison

\begin{tabular}{|llll|l|l|}
\hline Author & MAPE & MBE & nRMSE & MSE & MRE \\
\hline Our Results & $11.83-39.13 \%$ & $0.0242-19.354$ & $8.57-24.99 \%$ & - & - \\
Kardakos et al. (2013) & - & - & $3.17-20.35 \%$ & - & - \\
Cai et al. (2010) & $16.47-30.72 \%$ & - & - & - & - \\
Sharma et al. (2016) & - & - & - & 7.5 & - \\
Das et al. (2018) & $2.38-18.89 \%$ & - & $2.74-36.9 \%$ & - & $8.64-17.58 \%$ \\
\hline
\end{tabular}

\section{Conclusion}

With the increasing of solar energy in power generation, the power generated from a PV power plant need to be forecasted for operational planning, plant optimization, and energy trade. Several forecasting models exist; however, the choice of forecasting model depends on data availability, purpose, and needed resolution and accuracy. This study proposes a NARX model to forecast solar PV output power where global solar radiation, sunshine intensity, hourly temperature, minimum temperature, maximum temperature, wind speed, relative humidity, air pressure, and precipitation historical data are used as exogenous input. Hour angle and solar zenith angle of the location under consideration are also calculated and utilized. Additionally, the effects of changing the number of neurons in the hidden layer and increasing and decreasing the number of delays are also investigated.

The combination of input data was chosen depending on the regression values between each input parameter and the solar PV output power data. Three significant combinations of the nine meteorological variables are chosen and each combination is used with and without filling in for missing values. Hence, the impact of missing data to the performance of the model is considered. In total, six different data sets are used as input to the model and results are compared within data sets and across other studies. The performance of the NARX model is evaluated by using statistical evaluation parameters. The best performance, in general, is obtained by data set 3, where input data set is filled in for missing data, and wind speed and precipitation data are not used. The performance of the model is satisfactory, and the power can be predicted for hourly forecast with MAE, MAPE, MBE, and nRMSE errors of $52.0815,11.83 \%, 8.253$, and $8.57 \%$, respectively, and for daily forecast with $20.858,4.60 \%$, 9.206 , and $4.53 \%$, respectively.

This study shows the ability of NARX network in predicting solar PV plant produced power. The methodology demonstrates how to choose the best combination of data sets and how to deal with the missing data. Solar PV companies and researchers can use the methodology for the implementation of prediction models for solar PV output. Furthermore, our ongoing research on the subject focuses on machine learning algorithms for solar PV output power forecast and the results from both studies will be compared to determine the best approach.

\section{$\underline{\text { ORCID }}$}

O. Nkuriyingoma

S. B. Selçuklu
0000-0002-9637-8280

0000-0002-9295-3866

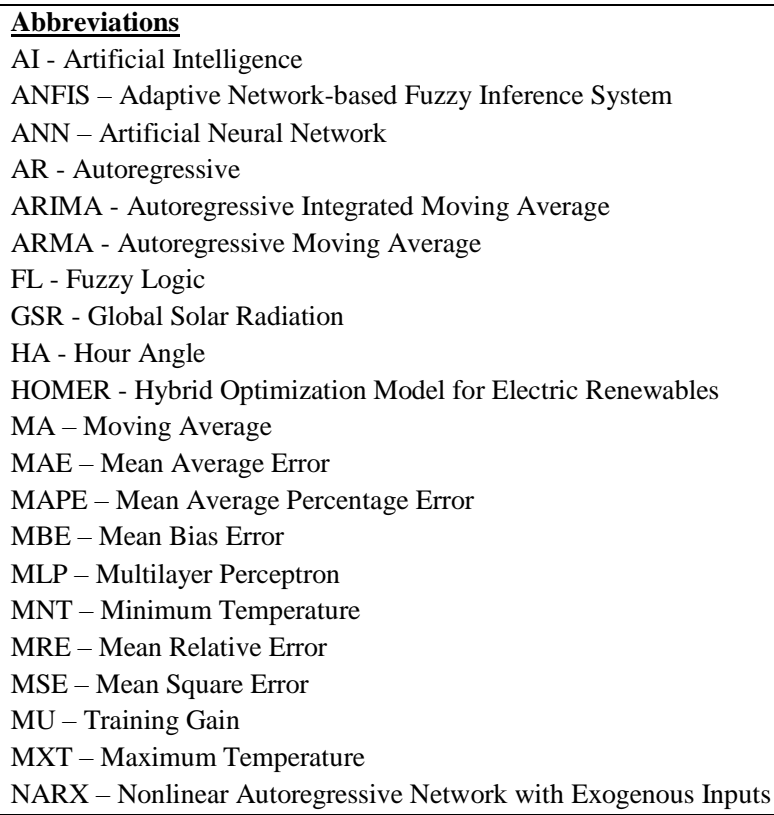

NMAE - Normalized Mean Absolute Error nRMSE - Normalized Root Mean Square Error NWP - Numerical Weather Prediction OUTP - Output Power

PRC - Precipitation

PRS - Air Pressure

PV - Photovoltaic

RBF- Radial Basis Function

RLH - Relative Humidity

SARIMA - Seasonal Autoregressive Integrated Moving Average RMSE - Root Mean Square Error

SSI - Sunshine Intensity

SVM - Support Vector Machine

TDL - Tapped Delay Lines

TMP - Hourly Temperature

TRNSYS - Transient Systems Simulation

UAE - United Arabic Emirates

WDS - Wind Speed

WMAE - Weighted Mean Absolute Error

WT - Wavelet Transform

ZA - Zenith Angle 


\section{References}

[1] Ayvazoğluyüksek, Ö. and Başaran, Filik Ü. 2018. Estimation methods of global solar radiation, cell temperature and solar power forecasting: A review and case study in Eskişehir. Renewable and Sustainable Energy Reviews, 91, 639-653.

[2] Das, U., Tey, K., Seyedmahmoudian, M., Mekhilef, S., Idris, M., Deventerc, W., Horan, B. and Stojcevski, A. 2018. Forecasting of photovoltaic power generation and model optimization: a review. Renewable and Sustainable Energy Reviews, 81, 912-928.

[3] Lorenz, E., Hurka, J., Heinemann, D. and Beyer, G. 2009. Irradiance forecasting for the power prediction of grid-connected photovoltaic systems. IEEE Journal of selected topics in applied earth observations and remote sensing, 2, 1-9.

[4] Ahmad, A., Anderson, T. and Lie, T. 2015. Hourly global solar irradiation forecasting for New Zealand. Solar Energy, 122, 1398-1408.

[5] Sharifzadeh, M., Sikinioti Lock, A. and Shah, N. 2019. Machine-learning methods for integrated renewable power generation: A comparative study of artificial neural networks, support vector regression, and gaussian process regression. Renewable and Sustainable Energy Reviews, 108, 513-538.

[6] Solangi, K., Islam, M., Saidur, R., Rahim, N. and Fayaz, H. 2010. A review on global solar energy policy. Renewable and sustainable energy reviews, 15, 21492163.

[7] Küçükdeniz, T. 2010. Long term electricity demand forecasting: An alternative approach with support vector machines. Istanbul University of Engineering Science, 1, 45:53.

[8] Frohlich, C. and Brusa, R. 1981. Solar radiation and its variation in time. Solar physics, 74, 209-215.

[9] Huang, R., Huang, T., Gadh, R. and Li, N. 2012. Solar generation prediction using the ARIMA model in a laboratory-level micro-grid. 2012 IEEE Third International conference on smart grid communications, 5-8 November, Tainan, Taiwan, 528533.

[10] Diagne, H.M., David, M., Lauret, P. and Boland, J. 2013. Review of solar irradiance forecasting methods and a proposition for small-scale insular grids. Renewable and Sustainable Energy Reviews, 27, 65-76.

[11] Wan, C., Zhao, J., Song, Y., Xu, Z., Lin, J. and Hu, Z. 2015. Photovoltaic and solar power forecasting for smart grid energy management. CSEE Journal of power and energy systems, 1, 38-46.

[12] Hong, W. Intelligent Energy Demand Forecasting. Springer, United Kingdom, 2013.
[13] Kardakos, E.G., Alexiadis, M.C., Vagropoulos, S.I., Simoglou, C.K., Biskas, P.N. and Bakirtzis, A.G. 2013. Application of time series and artificial neural network models in short-term forecasting of PV power generation. 2013 48th International Universities' Power Engineering Conference (UPEC), 2-5 September, Dublin, Ireland, 1-6.

[14] Basheer, I. and Hajmeer, M. 2000. Artificial neural networks: fundamentals, computing, design, and application. Journal of Microbiological Methods, 43, 331.

[15] Al Shamisi, M.H., Assi, A.H. and Hassan, A.N.H. Using Matlab to develop artificial neural network models for predicting global solar radiation in al Ain city - UAE. in: Assi, A.H., Engineering Education and Research Using MATLAB. Intech Publisher, 2011, 219238.

[16] Mazorra Aguiar, L., Pereira, B., David, M., Diaz, F. and Lauret, P. 2015. Use of satellite data to improve solar radiation forecasting with bayesian artificial neural networks. Solar Energy, 122, 1309-1324.

[17] Mohammed, L., Hamdana, M., Abdelhafeza, E. and Shaheenb, W. 2013. Hourly solar radiation prediction based on nonlinear autoregressive exogenous (narx) neural network. Jordan Journal of Mechanical and Industrial Engineering, 7, 11-18.

[18] Rao, K.D., Premalatha, M. and Naveen, C. 2018. Analysis of different combinations of meteorological parameters in predicting the horizontal global solar radiation with ANN approach: A case study. Renewable and Sustainable Energy Reviews, 91, 248-258.

[19] Yagli, G., Yang, D. and Srinivasan, D. 2019. Automatic hourly solar forecasting using machine learning models. Renewable and Sustainable Energy Reviews, 105, 487498.

[20] EL-Baz, W., Tzscheutschler, P. and Wagner, U. 2018. Day-ahead probabilistic PV generation forecast for buildings energy management systems. Solar Energy, $171,478-490$.

[21] Li, L., Weng, S., Tseng, M. and Wang, C. 2019. Renewable energy prediction: A novel short-term prediction model of photovoltaic output power. Journal of Cleaner Production, 228, 359-375.

[22] Li, L., Zhan, M. and Bai, Y. 2019. A recursive ensemble model for forecasting the power output of photovoltaic systems. Solar Energy, 189, 291-298.

[23] Pierro, M., De Ferice, M., Maggioni, E., Moser, D., Perotto, A., Spada, F. and Cornaro, C. 2018. Photovoltaic generation forecast for power transmission scheduling: A real case study. Solar Energy, 174, 976990. 
[24] Sharma, G., Pandey, A. and Chaudhary, P. 2016. Prediction of output solar power generation using neural network time series method. International Conference on Electrical Engineering (ICEENG), 1921 April, 10, Cairo, Egypt, 1-5.

[25] Sun, Y., Venugopal, V. and Brandt, A. 2019. Shortterm solar power forecast with deep learning: Exploring optimal input and output configuration. Solar Energy, $188,730-741$.

[26] Cadenas, E., Rivera, W., Amezcua, C. and Heard C. 2016. Wind speed prediction using a univariate ARIMA model and a multivariate narx model. Energies, 9, 1-15.

[27] DiPiazza, A., DiPiazza, M. and Vitale G. 2016. Solar and wind forecasting by NARX neural networks. Renewable Energy and Environmental Sustainability, $39,1-5$.

[28] Sandhya, T. and Kavitha, V. 2015. Estimation of solar radiation with various climatic parameters based on neural network. International Journal of Emerging Technology in Computer Science I\& Electronics (IJETCSE), 13, 151-155.

[29] Ahmad, A. and Anderson, T. 2014. Global solar radiation prediction using artificial neural network models for New Zealand. Australian Solar Energy Society (Australian Solar Council), 52, 141-150.

[30] Cai, T., Duan, S. and Chen, C. 2010. Forecasting power output for grid-connected photovoltaic power system without using solar radiation measurement. International Symposium on Power Electronics for Distributed Generation Systems, 2, 773-777.

[31] Palit, A. and Popovic, D. Computational Intelligence in Time Series Forecasting: Theory and Engineering Applications (Advances in Industrial Control). Springer, London, United Kingdom, 2005. 